\title{
Modified Hyperspheres Algorithm to Trace Homotopy Curves of Nonlinear Circuits Composed by Piecewise Linear Modelled Devices
}

\author{
H. Vazquez-Leal, ${ }^{1}$ V. M. Jimenez-Fernandez, ${ }^{1}$ B. Benhammouda, ${ }^{2}$ U. Filobello-Nino, ${ }^{1}$ \\ A. Sarmiento-Reyes, ${ }^{3}$ A. Ramirez-Pinero, ${ }^{1}$ A. Marin-Hernandez, ${ }^{4}$ and J. Huerta-Chua ${ }^{5}$ \\ ${ }^{1}$ Electronic Instrumentation and Atmospheric Sciences School, Universidad Veracruzana, Cto. Gonzalo Aguirre Beltrán S/N, \\ 91000 Xalapa, VER, Mexico \\ ${ }^{2}$ Higher Colleges of Technology, Abu Dhabi Men's College, P.O. Box 25035 Abu Dhabi, UAE \\ ${ }^{3}$ National Institute for Astrophysics, Optics and Electronics, Luis Enrique Erro No. 1, Santa María Tonantzintla, \\ 72840 Puebla, PUE, Mexico \\ ${ }^{4}$ Department of Artificial Intelligence, Universidad Veracruzana, Sebastián Camacho No. 5, 91000 Xalapa, VER, Mexico \\ ${ }^{5}$ Facultad de Ingeniería Civil, Universidad Veracruzana, Venustiano Carranza S/N, Colonia Revolución, \\ 93390 Poza Rica, VER, Mexico
}

Correspondence should be addressed to H. Vazquez-Leal; hvazquez@uv.mx

Received 22 April 2014; Accepted 2 July 2014; Published 11 August 2014

Academic Editor: Ishak Altun

Copyright (C) $2014 \mathrm{H}$. Vazquez-Leal et al. This is an open access article distributed under the Creative Commons Attribution License, which permits unrestricted use, distribution, and reproduction in any medium, provided the original work is properly cited.

\begin{abstract}
We present a homotopy continuation method (HCM) for finding multiple operating points of nonlinear circuits composed of devices modelled by using piecewise linear (PWL) representations. We propose an adaptation of the modified spheres path tracking algorithm to trace the homotopy trajectories of PWL circuits. In order to assess the benefits of this proposal, four nonlinear circuits composed of piecewise linear modelled devices are analysed to determine their multiple operating points. The results show that HCM can find multiple solutions within a single homotopy trajectory. Furthermore, we take advantage of the fact that homotopy trajectories are PWL curves meant to replace the multidimensional interpolation and fine tuning stages of the path tracking algorithm with a simple and highly accurate procedure based on the parametric straight line equation.
\end{abstract}

\section{Introduction}

The circuit simulation tools are constantly improved in order to cope with the challenges due to the new fabrication technologies. Among the circuit analysis methodologies, the direct current (DC) analysis is highlighted as one of the most important because it describes the static behaviour of the circuits. As a result of the DC analysis of nonlinear circuits, one obtains a nonlinear algebraic equations system (NAES). The most common method applied to solve such equations is the Newton-Raphson method (NRM). However, it is common that NRM fails due to its well-known problems of convergence: oscillation and divergence to infinity, among others. In fact, NRM has a local convergence only, which means that if the starting point is not close enough to the sought solution the method will probably diverge. What is more, if the circuit under analysis is multistable, then NRM will not be helpful because it can locate only one solution per simulation, ignoring the existence of more solutions. Therefore, the homotopy continuation method (HCM) [1-37] arises as an alternative to NRM due to its characteristics: to find multiple operating points and better convergence [38].

In recent years, the PWL modelling technique gained popularity as a tool for circuit simulation and other related areas $[39,40]$. The basic idea is to replace traditional models by their piecewise linear (PWL) representations [41-44]. The main advantages are reduction of equations complexity, the straightforward inclusion of empirical models, and potential 
replacing of piecewise models by their unified PWL representation. Several methodologies have been proposed to find multiple solutions of PWL circuits [45-57].

However such methodologies exhibit some drawbacks like the requirement of several initial points to find multiple solutions [53, 54], the use of implicit PWL models $[55,56]$, and the need of expressing the circuit equations in terms of the linear complementary problem (LCP) that implies computing model state variables [57]. Therefore, in order to circumvent the aforementioned disadvantages, we explore the application of HCM methods in combination with an adaptation of the modified spheres algorithm (MSA) [37] for the DC analysis of PWL circuits.

This paper is organized as follows. A brief description of PWL modelling is presented in Section 2. In Section 3, we introduce the proposed HCM and its path following technique (MSA). In Section 4, four case studies of nonlinear circuits are presented and solved by using a HCM method. Numerical simulations and a discussion about the results are provided in Section 5. Finally, a concluding remark is given in Section 6.

\section{Brief Description of PWL Modelling}

A mathematical model approach, widely used in nonlinear circuit analysis, is the so-called piecewise linear (PWL). The aim of this kind of modeling is to approximate the nonlinear behavior of a circuit element by using a set of linear mappings. This means transforming a single nonlinear equation into a finite number of linear equations. One of the first piecewise linear models was provided by Chua and Kang in [58]. Another proposal was presented by Van Bokhoven in [59]. Subsequent contributions were the extension of the Chua model reported by Guzelis and Goknar in [60] and the parametric proposal given by Vandenberghe et al. in [61], among others. While there are diverse proposals of PWL models, they can be classified into two classes. The first one contains explicit models. For this class of models, the output vector can be obtained by just substituting the input vector into the model. The second one contains models which are implicit. In such models the output vector cannot be obtained directly. In contrast, an algorithm has to be performed by which the output vector is computed [62]. The more representative examples of explicit and implicit PWL descriptions are the canonical model of Chual and the model of Bokhoven1, respectively.

The formal definition of the Chual model is expressed as follows.

Theorem 1. Any one-dimensional piecewise linear curve with $L$ segments and $\sigma$ break points $\beta_{1}<\beta_{2}<\cdots<\beta_{\sigma}$ can be represented by the expression

$$
y(x)=a+b x+\sum_{i=1}^{\sigma} c_{i}\left|x-\beta_{i}\right|,
$$

where the model parameters can be computed by

$$
\begin{gathered}
a=y(0)-\sum_{i=1}^{\sigma} c_{i}\left|\beta_{i}\right|, \\
b=\frac{J^{(1)}+J^{(\sigma+1)}}{2}, \\
c_{i}=\frac{J^{(i+1)}-J^{(i)}}{2}, \quad i=1,2, \ldots, \sigma,
\end{gathered}
$$

with $J^{(i)}$ denoting the slope of the ith constitutive segment in the piecewise linear curve.

Meanwhile, the Bokhovenl model is expressed by a state variable system defined in formulation of LCP. For further details about LCP, the reader is referred to [63].

The main factor that motivates the use of PWL models is the simplicity of their structure, which is linear in each region of the domain. However, in terms of circuit analysis the use of piecewise linear models means transforming a single nonlinear equation into several linear equations that could easily be solved by standard methods from linear algebra. The problem lies now in the extremely large number of linear regions to be discarded to determine the entire set of circuit solutions. Unfortunately, this task requires enormous computational resources. To overcome that problem several methodologies and algorithms have been proposed. For example, Chua and Ying [64] reported an efficient method where the number of linear simultaneous equations to be solved could be decreased by a sign test. The same idea is improved by Yamamura and Ochiai in [65] where linear programming techniques are applied and a more efficient sign test algorithm is also reported. Katzenelson presents an algorithm based on Newton's homotopy in [53], and more recently Tadeusiewicz and Kuczyński offered a method that combines the homotopy concept and the theory known as a linear complementary problem [57].

\section{The Proposed Homotopy Scheme}

The equilibrium equation to describe the DC behaviour is obtained using the Kirchhoff laws, resulting in

$$
\mathbf{f}(\mathbf{x})=\mathbf{0}, \quad \mathbf{f} \in \mathfrak{R}^{n} \longrightarrow \mathfrak{R}^{n},
$$

where $\mathbf{x}$ represents the electrical variables of the circuit and $n$ the number of variables.

Homotopy methods are based on the fact that solutions are connected by a curve denominated "solution curve" or "homotopy curve." Such curve is induced by including an extra parameter in the original NAES, resulting in

$$
\mathbf{H}(\mathbf{f}(\mathbf{x}), \lambda)=0, \quad \mathbf{H} \in \mathfrak{R}^{n} \times \mathfrak{R} \longrightarrow \mathfrak{R}^{n},
$$

where $\lambda$ is the homotopy parameter and $\mathbf{H}^{-1}(\mathbf{0})$ the family of solutions that conforms the homotopy path.

An example of homotopy formulation is Newton's homotopy

$$
\mathbf{H}(\mathbf{f}(\mathbf{x}), \lambda)=\mathbf{f}(\mathbf{x})+(\lambda-1) \mathbf{f}\left(\mathbf{x}_{i}\right)=\mathbf{0},
$$

where $\mathbf{x}_{i}$ is the starting point of the trajectory. 


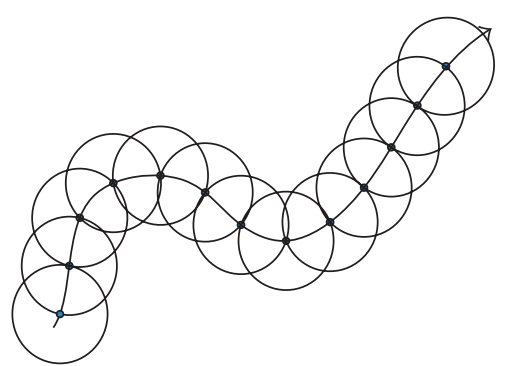

(a) General homotopy curve

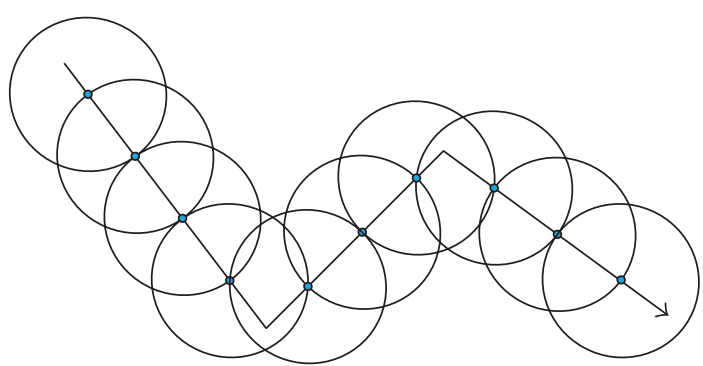

(b) Piecewise linear homotopy curve

FIGURE 1: Solution curves with spheres [37].

This system has the following properties.

(1) At the starting point $\lambda=0$,

$$
\mathbf{H}(\mathbf{f}(\mathbf{x}), 0)=\mathbf{f}(\mathbf{x})-\mathbf{f}\left(\mathbf{x}_{i}\right)=\mathbf{0},
$$

where the homotopy system admits at least the solution $\mathbf{x}_{i}$.

(2) The deformation continues until crossing $\lambda=1$ where

$$
\mathbf{H}(\mathbf{f}(\mathbf{x}), 1)=\mathbf{f}(\mathbf{x})=\mathbf{0}
$$

that is, the homotopy is reduced to (3).

Thus, the original problem becomes a numerical continuation problem $[4,5,12,13,21,25-28]$, where the continuation variable is the homotopy parameter $\lambda$. The homotopy map creates a continuous line that crosses several times $\lambda=1$ depending on the number of operating points. A drawback of the homotopy methods is that there is no generalized methodology to guarantee that a single homotopy path possesses all the operating points of any given nonlinear circuit. In contrast, HCM can locate multiple operating points in comparison to NRM that can fail to find even a single operating point.

3.1. Modified Spheres Algorithm. Once the equilibrium equation and homotopy map are constructed, a new problem emerges: the homotopy trajectory should be traced in order to detect the roots. It is well known from the literature that if the path tracking algorithm is not correctly implemented, the simulation may fail to detect any root even though the roots are, in fact, along the curve $[4,5,12,13,21,25-28]$. For the case of PWL circuits, the problem for the path tracking algorithm lies in the prediction stage, because most of the predictor mechanisms are based on the tangent of the homotopy curve. If we consider that the derivative of PWL functions is not defined at the break points, then the tangent of the homotopy curve can not be evaluated at such points. Therefore, we propose adapting the modified spheres algorithm (MSA) for the path following of the homotopy curves of PWL circuits, which is not based on the use of tangents of the trajectory.

The homotopy formulation contains $n$ equations and $(n+1)$ variables, where $x_{i}(i=1, \ldots, n)$ represent the variables of the system and $x_{n+1}$ is the homotopy parameter $\lambda$. Nevertheless, if we add the equation that describes a sphere $[2,3,13,37,66]$ with center at $c$ (initial point of the trajectory) and radius $r$ expressed by

$$
\begin{aligned}
S\left(x_{1}, x_{2}, \ldots, x_{n+1}\right)= & \left(x_{1}-c_{1}\right)^{2}+\left(x_{2}-c_{2}\right)^{2} \\
& +\cdots+\left(x_{n+1}-c_{n+1}\right)^{2}-r^{2}=0,
\end{aligned}
$$

then, it is possible to apply a regular NRM to solve the homotopy formulation.

Therefore, using (4) and (8), we formulate the augmented system as

$$
\begin{aligned}
& H_{1}\left(f_{1}(x), \lambda\right)=0, \\
& H_{2}\left(f_{2}(x), \lambda\right)=0, \\
& \vdots \\
& H_{n}\left(f_{n}(x), \lambda\right)=0, \\
& S\left(x_{1}, x_{2}, \ldots, x_{n}, \lambda\right)=0 .
\end{aligned}
$$

The solution curve can be traced by solving (9) for each hypersphere and updating the center of the hypersphere in each iteration step. The hyperspheres $\left(S_{1}, S_{2}, \ldots\right)$ are allocated successively as shown in Figure 1(a); at each step the solution obtained is used as the center of the new sphere. In the same fashion, Figure 1(b) depicts the application of MSA algorithm for the path tracking of PWL curves.

The proposed adaptation of the MSA scheme [37] for the Newton homotopy applied to PWL circuits is described as follows.

(i) Predictor: we use points $\mathrm{O}_{1}$ and $\mathrm{O}_{2}$ to predict the point $k_{1}$. The next predictor point and successive points are obtained as depicted in Figure 2(a).

(ii) Corrector: after calculating the point predictor $\left(k_{1}\right)$, a corrected point $\left(\mathrm{O}_{3}\right)$ is calculated by solving (9). This procedure is detailed in [37]. Nonetheless, if we consider that-for this work-the homotopy trajectory is described as a PWL curve, then the corrector step will require most of the time one iteration to correct the prediction over straight lines, except at the break points, where it will require more steps to correct the curve (see Figure 2(b)). 


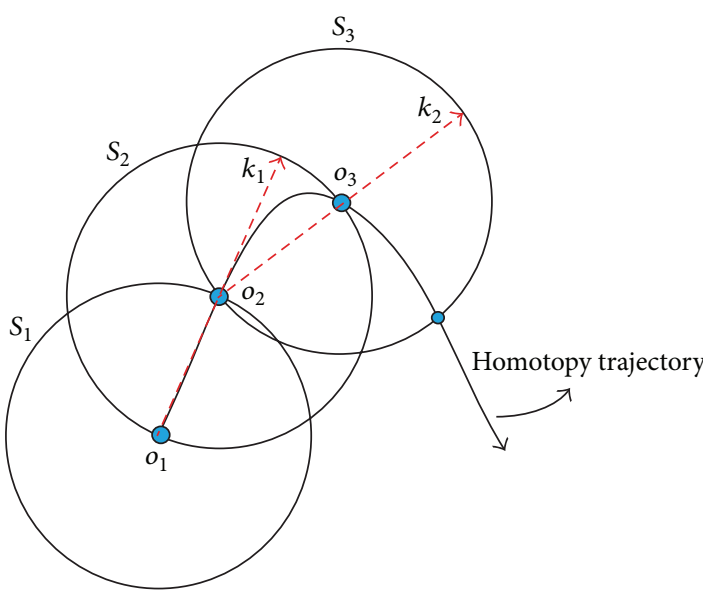

(a) General homotopy trajectory

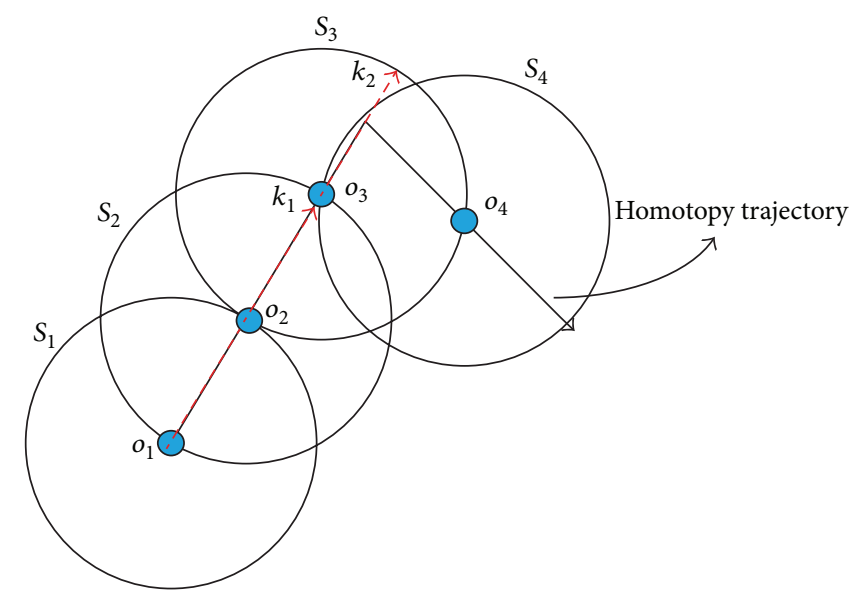

(b) Piecewise linear homotopy trajectory

FIGURE 2: Spheres algorithm [37].

(iii) There is a potential issue called reversion phenomenon that provokes a backward tracing. In [37] a strategy based on gradients and angles of the intersection of the sphere along the trajectory is proposed.

(iv) Find zero strategy $[12,22]$ : the finding zero strategy should start after the trajectory crosses $\lambda=1$. This procedure requires detecting the two points ( $A$ and $B$ ) before and after $\lambda=1$ as depicted in Figure 3 .

(v) Interpolation of operating points $[12,22]$ : traditional schemes of path tracking algorithms require the application of complicated multidimensional interpolation algorithms as those reported in [37]. Nonetheless, as we will show in the cases study section, the homotopy trajectory of PWL circuits is also a PWL curve. Therefore, we propose using the formula of a parametric straight line to interpolate the solution at $\lambda=1$. Using the points $A$ and $B$, we create two vectors $\mathbf{A}$ and $\mathbf{B}$, respectively, resulting in the following equation:

$$
\mathbf{B}+t(\mathbf{B}-\mathbf{A})=\mathbf{0},
$$

where $t$ is the parameter that describes the $n+1$ dimensional straight line. To perform the interpolation, we obtain the value of $t$ that induces $\lambda=1$ and update the rest of the equations to obtain the sought solution $S_{*}$ (see Figure 3 ). This process can be repeated each time the homotopy trajectory crosses $\lambda=1$.

(vi) Improving accuracy for final solutions also known as fine tuning [22]: traditional path following schemes including the ones reported for the MSA scheme $[13,37]$ require extra steps of NRM to improve the accuracy of the interpolated solutions. However, the aforementioned interpolation step can theoretically obtain a highly accurate solution. The reason relies on the fact that the homotopy curve crosses exactly over the roots of the equilibrium equation; then, the straight line (10) also crosses over the exact solution.

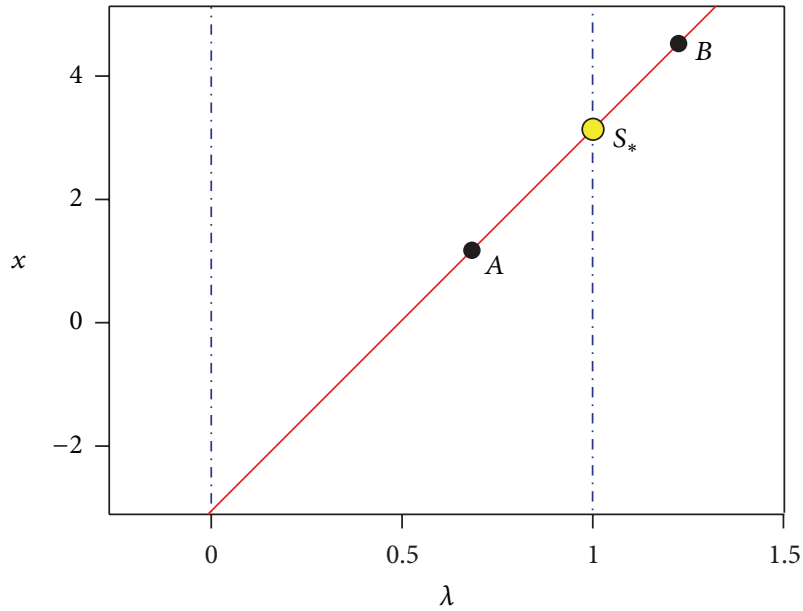

FIGURE 3: Interpolation procedure using a parametric straight line.

\section{Cases Study}

In the present section, we will solve four case studies [64] to show the usefulness of the proposed method to perform the DC analysis of nonlinear circuits composed of devices modelled using the explicit PWL model (1). For all the cases' study, we use a constant radius $r=0.1$ for the hyperspheres.

4.1. Circuit with Two Nonlinear Resistors. The following case study shows a simple circuit composed of two nonlinear resistors as depicted in Figure 4. The models of the resistors $R_{1}$ and $R_{2}$ are

$$
\begin{aligned}
R_{1}: i_{1}= & -\frac{125}{8}+\frac{9}{8} v_{1}+\frac{7}{8}\left|v_{1}+1\right|-\frac{3}{2}\left|v_{1}-2\right|+\frac{3}{4}\left|v_{1}-5\right| \\
& -\frac{1}{8}\left|v_{1}-11\right|-\frac{9}{8}\left|v_{1}-13\right|+2\left|v_{1}-15\right|,
\end{aligned}
$$


TABLE 1: Numerical solutions for (12).

\begin{tabular}{lcccc}
\hline Solution & Iteration & $v_{1}$ & $v_{2}$ & MSE $=\left(f_{1}^{2}+f_{2}^{2}\right) / 2$ \\
\hline$S_{1}$ & 168 & 1.49999999999 & 1.49999999998 & 0 \\
$S_{2}$ & 196 & 4.00000000122 & 0.999999999476 & $2.22 e-18$ \\
$S_{3}$ & 214 & 5.66666666670 & 0.666666666664 & $2.12 e-20$ \\
\hline
\end{tabular}

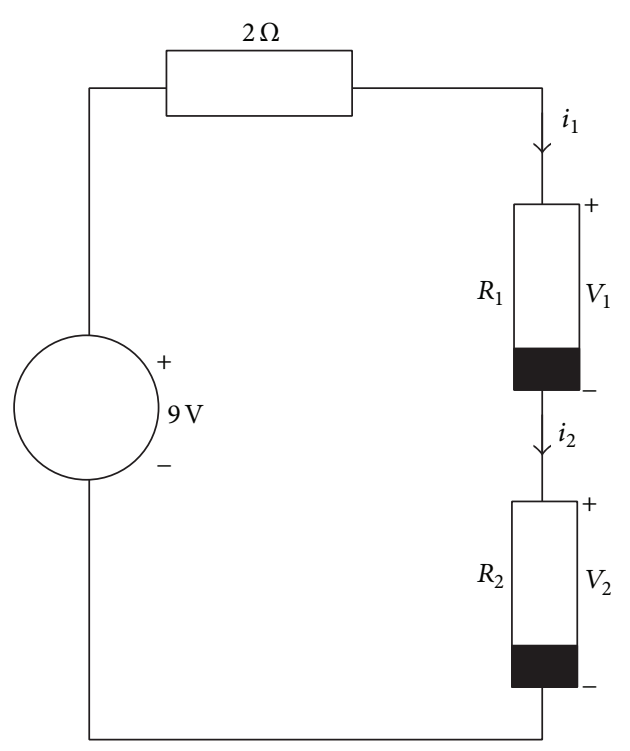

FIGURE 4: Two nonlinear resistor circuits.

$$
\begin{aligned}
R_{2}: i_{2}= & \frac{29}{4}+\frac{3}{2} v_{2}-\frac{3}{2}\left|v_{2}+8\right|+\frac{3}{2}\left|v_{2}+5\right|-\frac{3}{2}\left|v_{2}+3\right| \\
& +\frac{3}{2}\left|v_{2}+1\right|-\frac{3}{4}\left|v_{2}-3\right|-\frac{5}{4}\left|v_{2}-8\right|+\frac{3}{2}\left|v_{2}-10\right| \\
& +\left|v_{2}-13\right|-\frac{5}{4}\left|v_{2}-16\right|+\frac{1}{4}\left|v_{2}-18\right|,
\end{aligned}
$$

described by 7 and 11 PWL segments, respectively.

Using Kirchhoff laws, we obtain

$$
\begin{aligned}
& f_{1}\left(v_{1}, v_{2}\right)=v_{1}+v_{2}+2 i_{1}-9=0, \\
& f_{2}\left(v_{1}, v_{2}\right)=v_{1}+v_{2}+2 i_{2}-9=0 .
\end{aligned}
$$

Applying the Newton homotopy to (12) combined with MSA yields

$$
\begin{aligned}
& H_{1}\left(v_{1}, v_{2}, \lambda\right)=f_{1}\left(v_{1}, v_{2}\right)+(\lambda-1) f_{1}\left(v_{1,0}, v_{2,0}\right)=0, \\
& H_{2}\left(v_{1}, v_{2}, \lambda\right)=f_{2}\left(v_{1}, v_{2}\right)+(\lambda-1) f_{2}\left(v_{1,0}, v_{2,0}\right)=0, \\
& \quad S\left(v_{1}, v_{2}, \lambda\right)=\left(v_{1}-c_{1}\right)^{2}+\left(v_{2}-c_{2}\right)^{2}+\left(\lambda-c_{3}\right)^{2}-r^{2}=0,
\end{aligned}
$$

where $v_{1,0}=-5$ and $v_{2,0}=-4$ are the initial point of the homotopy at $\lambda_{0}=0$ and $S\left(v_{1}, v_{2}, \lambda\right)$ is the equation of the hypersphere whose center will be updated at each iteration of the method.
For the first hypersphere the center is located at $c_{1}=v_{1,0}$, $c_{2}=v_{2,0}$, and $c_{3}=\lambda_{0}$. The centers of the successive hyperspheres are obtained using the aforementioned procedure in Section 3.1. As a result of MSA algorithm, the three operating points of the circuit have been located (see Figure 5). In addition, Table 1 shows the computed solutions, iterations, and the mean square error (MSE).

4.2. Circuit with Three Nonlinear Resistors. The following case study shows a circuit composed of three nonlinear resistors as depicted in Figure 6. The models of $R_{1}, R_{2}$, and $R_{3}$ resistors are

$$
\begin{aligned}
& R_{1}: i_{1}=\frac{5}{6}\left|v_{1}+6\right|-\frac{5}{6}\left|v_{1}-6\right|, \\
& R_{2}: v_{2}=\frac{1}{6}\left|i_{2}+1\right|-\frac{1}{6}\left|i_{2}-5\right|, \\
& R_{3}: i_{3}=v_{3}-\frac{5}{4}\left|v_{3}-1\right|+2\left|v_{3}-2\right|-\left|v_{3}-3\right|,
\end{aligned}
$$

described by 3, 3, and 4 PWL segments, respectively.

Using Kirchhoff laws [64], we obtain

$$
\begin{aligned}
& f_{1}\left(v_{1}, v_{2}, v_{3}\right)=v_{1}+i_{2}+v_{3}-i_{1}-5=0, \\
& f_{2}\left(v_{1}, v_{2}, v_{3}\right)=i_{2}+v_{3}-v_{2}-5=0, \\
& f_{3}\left(v_{1}, v_{2}, v_{3}\right)=-v_{3}-i_{3}+5=0 .
\end{aligned}
$$

Next, we apply the Newton homotopy to (15) as done for the first case study, using $v_{1,0}=15, i_{2,0}=-1$, and $v_{3,0}=15$ as the initial point of the homotopy. As a result of tracing the homotopy path, the three operating points of the circuit have been located (see Figure 7). In addition, Table 2 shows the found solutions, iterations, and the mean square error (MSE).

4.3. Schmitt Trigger Circuit. Consider the Schmitt trigger circuit of Figure 8(a), where the bipolar transistors are modelled using the simplified Ebers-Moll (see Figure 8(b)) model of NPN transistors as depicted in Figure 8(c). The PWL model of five segments of the diodes of all transistors is

$$
\begin{aligned}
i_{d}\left(v_{d}\right)= & -0.05486777833+0.1482755558 v_{d} \\
& +0.01157779318\left|v_{d}-0.306\right| \\
& +0.01181869788\left|v_{d}-0.3375\right| \\
& +0.04904536922\left|v_{d}-0.366\right| \\
& +0.07583369515\left|v_{d}-0.3875\right|
\end{aligned}
$$


TABLE 2: Numerical solutions for (15).

\begin{tabular}{lccccc}
\hline Solution & Iteration & $v_{1}$ & $i_{2}$ & $v_{3}$ & MSE \\
\hline$S_{1}$ & 137 & 9.9333333333 & 2.20000000000 & 2.866666666667 & $1.71 e-22$ \\
$S_{2}$ & 382 & 0.0999999999 & 2.20000000000 & 2.86666666666 & $1.45 e-22$ \\
$S_{3}$ & 610 & -10.0666666666 & 2.20000000000 & 2.86666666667 & $3.86 e-23$ \\
\hline
\end{tabular}

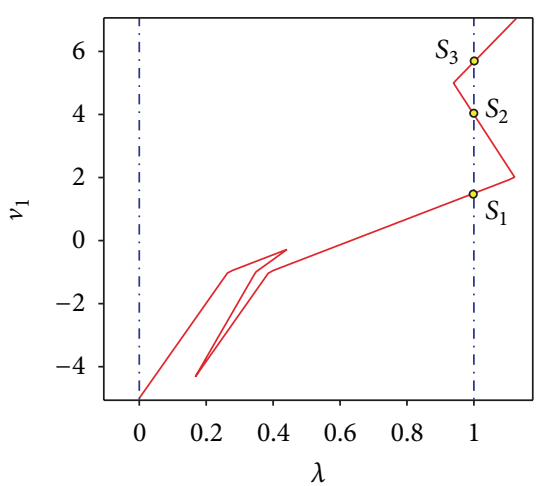

(a) Projection $\lambda$ - $v_{1}$

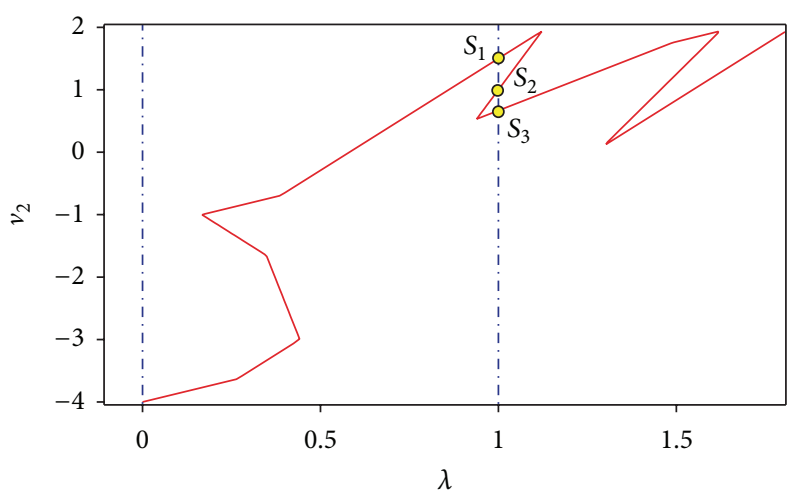

(b) Projection $\lambda-v_{2}$

Figure 5: Homotopy path for (13).

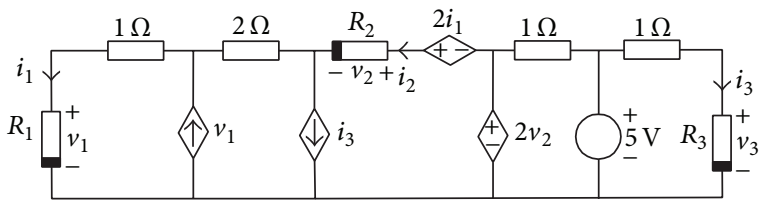

FIGURE 6: Three nonlinear resistor circuits.

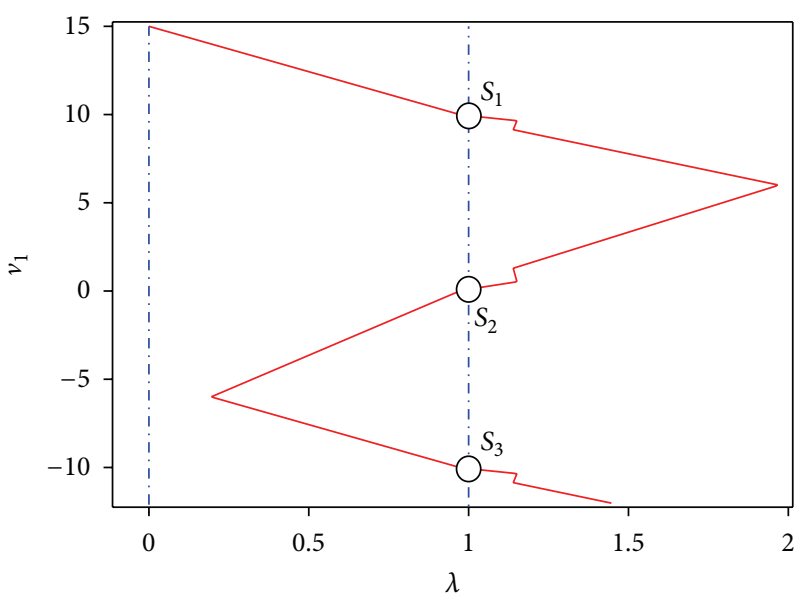

FIgURE 7: Projection $\lambda-v_{1}$ of homotopy path for (15).

Using Kirchhoff laws, we obtain

$$
\begin{aligned}
& f_{1}\left(v_{1}, v_{2}\right)=3.33-1000\left(i_{d}\left(v_{1}\right)+i_{d}\left(v_{2}\right)\right)-v_{1}=0, \\
& f_{2}\left(v_{1}, v_{2}\right)=4-1392 i_{d}\left(v_{1}\right)-1096 i_{d}\left(v_{2}\right)-v_{2}=0 .
\end{aligned}
$$

Then, Newton homotopy is applied in the same fashion as in the first example, using as starting point $v_{1,0}=-5$ and $v_{2,0}=-2$ at $\lambda=0$. The results show that the homotopy trajectory crosses for the three operating points of the Schmitt trigger circuit as depicted in Figure 9 and Table 3.

4.4. Chua's Circuit with Nine Solutions. Consider Chua's circuit of Figure 10, where the bipolar transistors are modelled using the simplified Ebers-Moll (see Figure 8(b)) model of NPN transistors. The PWL model for the diodes of all transistors is (16).

Using Kirchhoff laws, we obtain

$$
\begin{aligned}
f_{1}\left(v_{1}, v_{2}, v_{3}, v_{4}\right)= & 4.36634 v_{2}+6103.168 i_{d}\left(v_{1}\right) \\
& +2863.168 i_{d}\left(v_{2}\right)-12=0, \\
f_{2}\left(v_{1}, v_{2}, v_{3}, v_{4}\right)= & 5.4 v_{1}+v_{3}+3580 i_{d}\left(v_{1}\right)+6620 i_{d}\left(v_{2}\right) \\
& +700 i_{d}\left(v_{3}\right)+500 i_{d}\left(v_{4}\right)-22=0, \\
f_{3}\left(v_{1}, v_{2}, v_{3}, v_{4}\right)= & 4.36634 v_{4}+6103.168 i_{d}\left(v_{3}\right) \\
& +2863.168 i_{d}\left(v_{4}\right)-12=0, \\
f_{4}\left(v_{1}, v_{2}, v_{3}, v_{4}\right)= & v_{1}+5.4 v_{3}+700 i_{d}\left(v_{1}\right) \\
& +3580 i_{d}\left(v_{3}\right)+6620 i_{d}\left(v_{4}\right)-22=0 .
\end{aligned}
$$

The Newton homotopy is applied to (18) in the same way as in the first case study. We trace two trajectories with the following starting points: $Q_{1}=[-7,-1,8,1]$ and $Q_{2}=$ $[0,-7,0,0]$. After using the adapted MSA algorithm, the nine solutions of the circuit were found (see Figure 11). In addition, Table 4 shows the found solutions, iterations, and the mean square error (MSE). 
TABLE 3: Numerical solutions for (17).

\begin{tabular}{lcccc}
\hline Solution & Iteration & $v_{1}$ & $v_{2}$ & MSE \\
\hline$S_{1}$ & 59 & 0.022145787125 & 0.374592098924 & $6.11 e-18$ \\
$S_{2}$ & 63 & 0.348923957274 & 0.358608185920 & $1.70 e-21$ \\
$S_{3}$ & 68 & 0.372176159314 & -0.117291118129 & $5 e-21$ \\
\hline
\end{tabular}

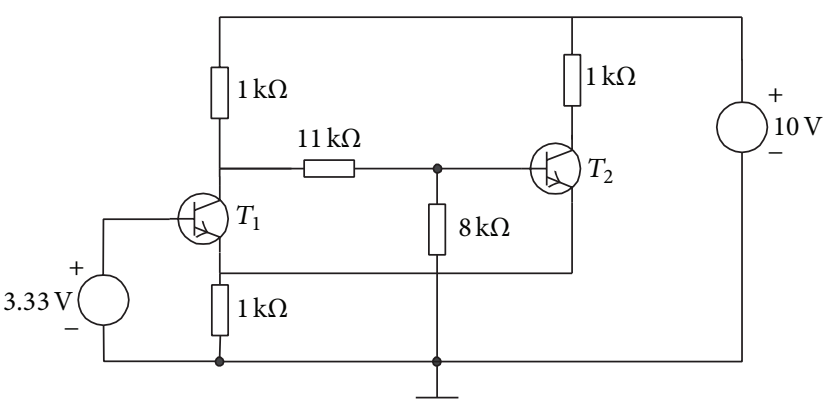

(a) Original circuit

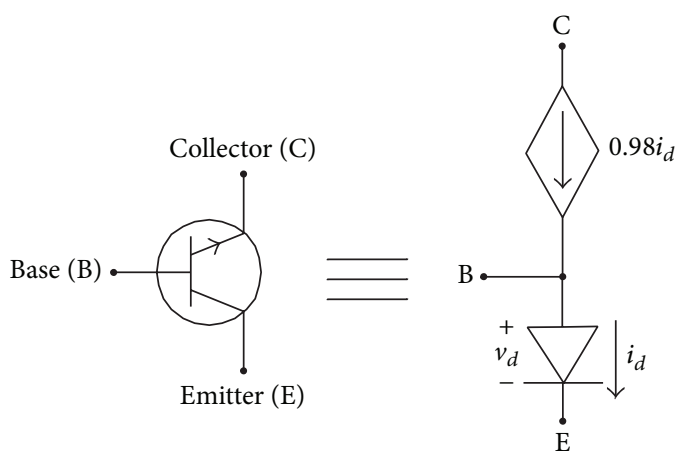

(b) Simplified Embers-Moll model

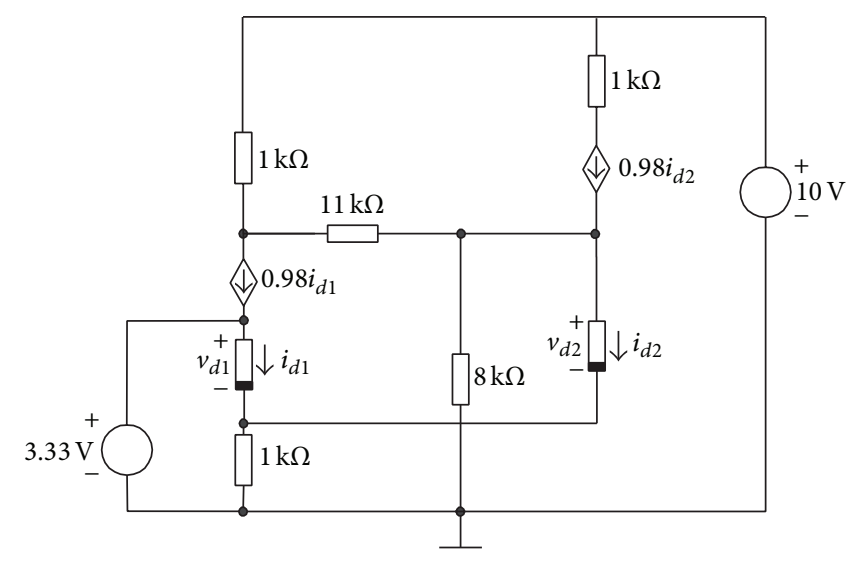

(c) Schmitt trigger circuit using Embers-Moll model

FIGURE 8: Schmitt trigger circuit.

\section{Numerical Simulation and Discussion}

All case studies were successfully solved using the proposed methodology. For the first three case studies, it was possible to find within a single trajectory the three operating points of each problem, and for the last case study, we find the nine solutions of Chua's circuit using two starting points. The high accuracy of the located operating points shows that the simple interpolation algorithm based on straight lines is a powerful tool and is simple to implement (see Tables 1-4). Besides, the accuracy of the interpolate solutions allows us to discard the stage of applying NRM extra steps to increase accuracy usually required by path tracking algorithms $[4,5$, $12,13,21,25-28]$. It is important to remark that the variety of solved circuits exhibits the high potential of HCM combined with MSA to solve multistable nonlinear circuits integrated by devices modelled with explicit PWL representations.

In $[53,54]$ methods based on the Newton homotopy are reported, which are capable of locating only one solution per simulation. Therefore if user requires to find more solutions, it is necessary to propose some random initial points to perform more simulations. Instead, the proposed methodology is capable of locating multiple operating points within a single path or simulation.

Methods reported in $[55,56]$ use implicit PWL models. This implies that the number of linear regions explodes due to the diode synthesis. Besides, compared to the explicit models, implicit PWL models require a more complex algorithm to compute the model state variables. The proposed methodology uses an explicit model representation easy to implement.

In [57] a methodology that depends on the specific circuit topology description of multiport with extracted ideal diodes is reported. In such methodology, circuit equations are expressed in terms of the LCP which implies computing model state variables. The proposed methodology is based on a straightforward methodology based on the traditional circuit analysis tools used to build commercial circuit simulators and a simple path tracking algorithm easy to implement. 
TABLE 4: Numerical solutions for (18).

\begin{tabular}{|c|c|c|c|c|c|c|c|}
\hline Path & Solution & Iteration & $v_{1}$ & $v_{2}$ & $v_{3}$ & $v_{4}$ & MSE \\
\hline$Q_{1}$ & $S_{1}$ & 105 & -0.734973033383 & 0.376723496304 & 0.318166142629 & 0.372621919721 & $1.14 e-17$ \\
\hline$Q_{1}$ & $S_{2}$ & 111 & -0.650249656974 & 0.376723495848 & -0.239265377696 & 0.376723494677 & $6.64 e-14$ \\
\hline$Q_{1}$ & $S_{3}$ & 235 & 0.326931131382 & 0.369666979551 & -0.48305281023 & 0.376723495005 & $3.61 e-16$ \\
\hline$Q_{1}$ & $S_{4}$ & 243 & 0.329725453371 & 0.368724929946 & 0.324331786706 & 0.370543296483 & $6.27 e-19$ \\
\hline$Q_{1}$ & $S_{5}$ & 312 & 0.386520101863 & -4.29094430670 & 0.337714498625 & 0.365872023364 & $6.40 e-17$ \\
\hline$Q_{1}$ & $S_{6}$ & 328 & 0.388146243604 & -4.75726420472 & -1.12762657849 & 0.376723498380 & $5.69 e-17$ \\
\hline$Q_{2}$ & $S_{7}$ & 58 & 0.383283219902 & -3.63542706051 & 0.383283217035 & -3.63542647848 & $1.73 e-16$ \\
\hline$Q_{2}$ & $S_{8}$ & 193 & 0.338139358469 & 0.364994969864 & 0.387969158453 & -4.68386026807 & $2.86 e-19$ \\
\hline$Q_{2}$ & $S_{9}$ & 215 & -1.19554608083 & 0.376723498781 & 0.389904592568 & -5.48612114477 & $2.42 e-16$ \\
\hline
\end{tabular}

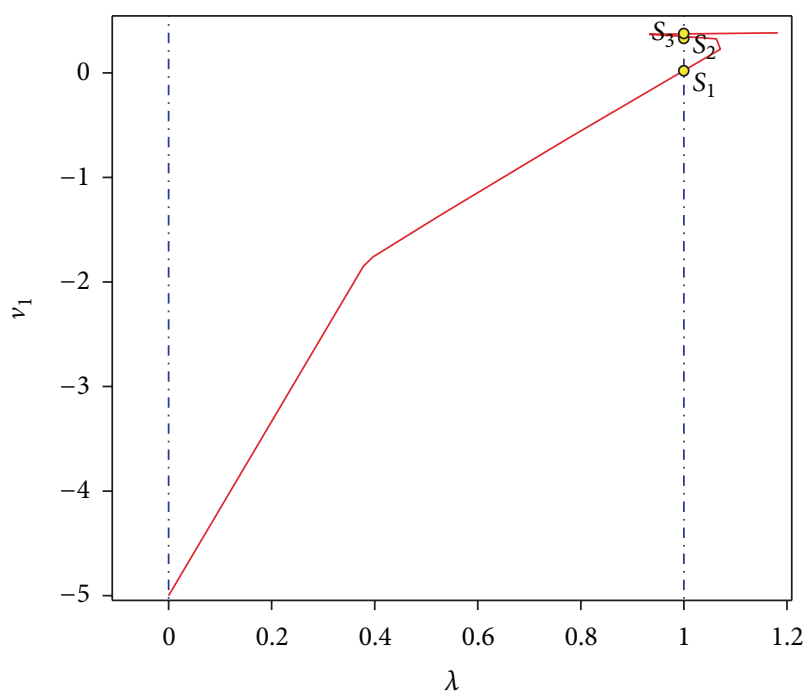

(a) Homotopy path

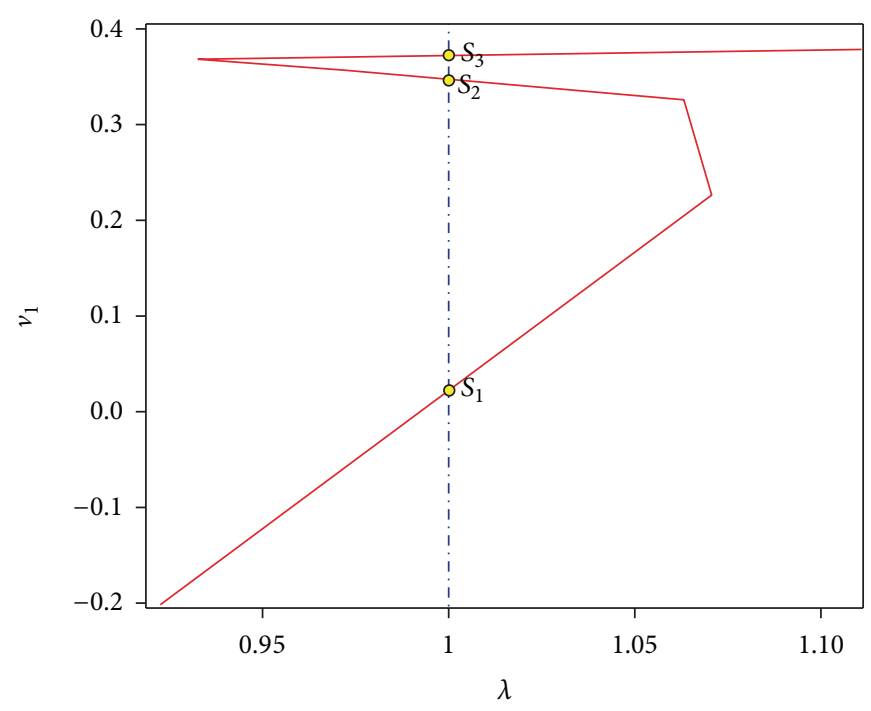

(b) Zoom to the solutions region

FIGURE 9: Homotopy path for (17) projected over $\lambda-v_{1}$.

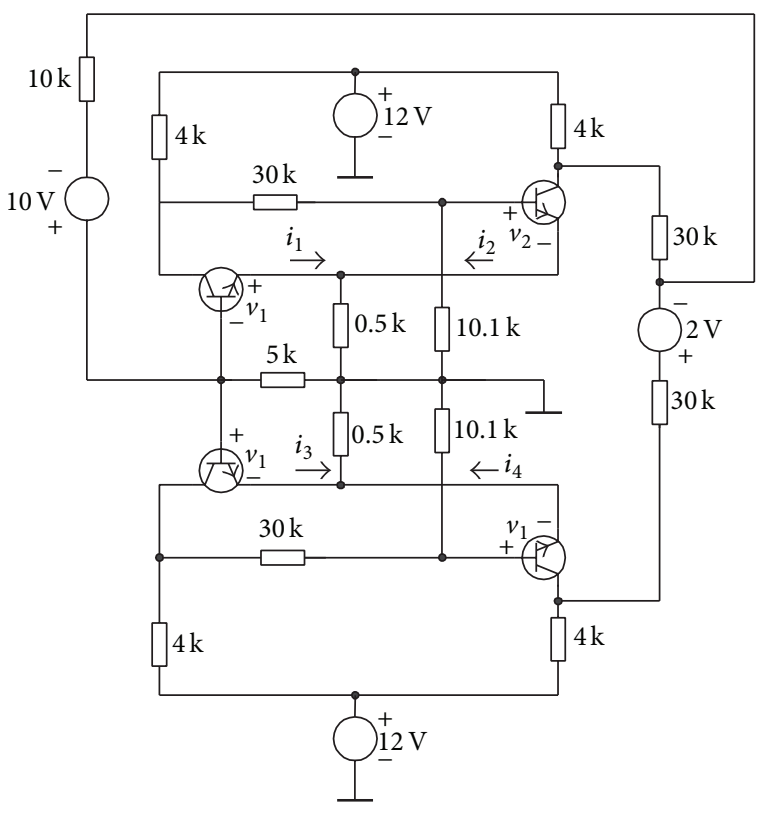

FIGURE 10: Chua's circuit with nine solutions.
Further research should be addressed in the following topics.

(i) Implement a strategy to use the fact that the homotopy curves are straight lines to accelerate the homotopy simulation.

(ii) Implement a circuit simulator to solve high density transistor circuits modelled by the PWL technique.

(iii) Replace the Newton homotopy by other methods as the fixed point homotopy [14], double bounded homotopy [12, 37], double bounded polynomial homotopy [11, 36], Newton fixed-point homotopy [67], $d$-homotopy [68], and multiparameter homotopy $[13,17]$, among others. This research can lead to proposal of better homotopy schemes with better results in aspects like number of found solutions, CPU time, and global convergence, among others.

(iv) Theoretically obtain the position of the break points of the PWL homotopy curve, significantly decreasing the number of steps. Such research can conduct to a very fast path tracking scheme. 


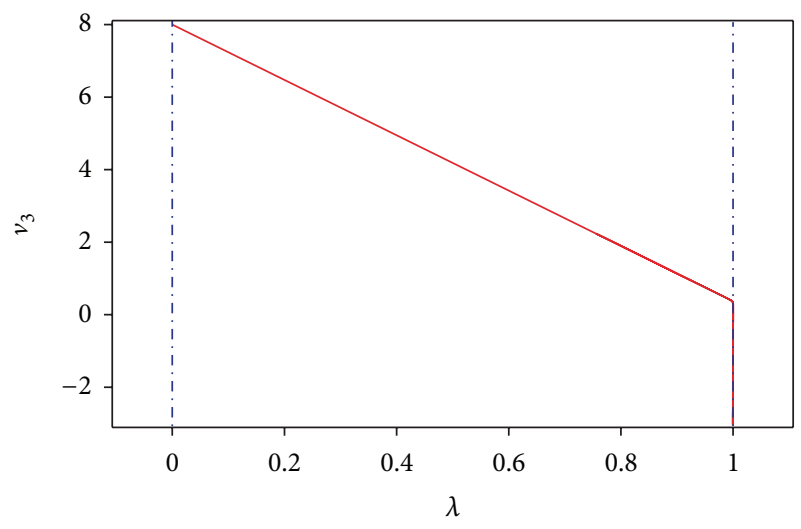

(a) Homotopy path for initial point $Q_{1}$

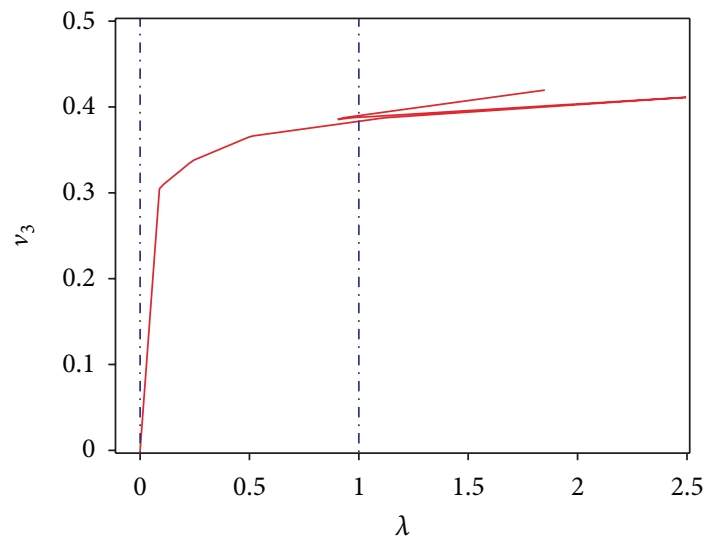

(c) Homotopy path for initial point $Q_{2}$

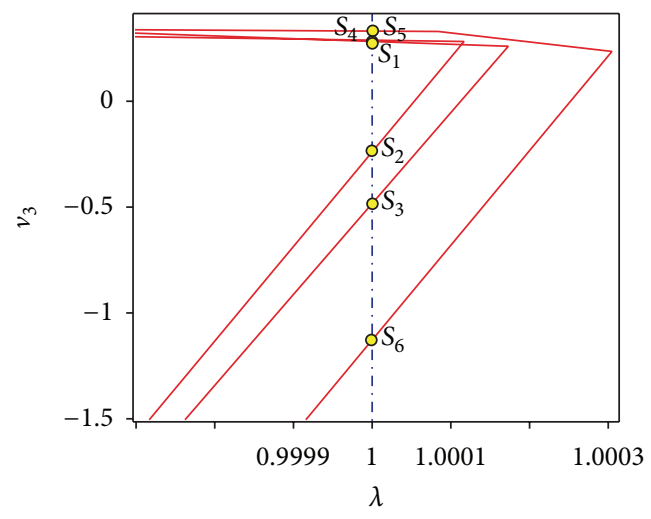

(b) Zoom for (a)

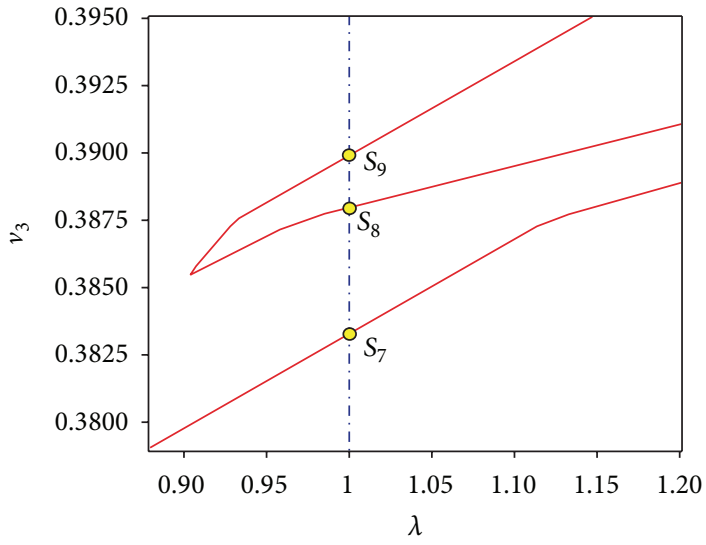

(d) Zoom for (c)

FIGURE 11: Homotopy paths for (18) projected over $\lambda-v_{3}$.

(v) Propose a methodology to obtain an optimal initial point for the homotopy simulation. This golden starting point will possess the characteristic of producing a minimum number of iterations and a maximum number of found solutions or all solutions.

\section{Conclusions}

In this work, we presented a homotopy scheme based on the Newton homotopy and a modified MSA path tracking algorithm, applied to the DC simulation of nonlinear circuits composed of devices modelled by PWL techniques. The effectiveness and power of the proposed scheme were exhibited by the successful solution of all the operating points of several circuits including devices as nonlinear resistors, diodes, transistors, and transactors, among others. In addition, the high accuracy of the solutions was reached by applying a simple interpolation technique that discards the use of Newton-Raphson extra steps to increase the accuracy of the interpolated solutions. Finally, further research should be performed to extend the application of the proposed scheme to very large integrated circuits (VLSI).

\section{Conflict of Interests}

The authors declare that there is no conflict of interests regarding the publication of this paper.

\section{Acknowledgments}

The authors would like to acknowledge Alejandra DiazArmendariz, Roberto Ruiz-Gomez, and Rogelio-Alejandro Callejas-Molina for their contribution to this project. $\mathrm{H}$. Vazquez-Leal gratefully acknowledges the financial support provided by the National Council for Science and Technology of Mexico (CONACyT) through Grant CB-2010-01 \#157024.

\section{References}

[1] H. Jimenez-Islas, G. M. Martinez-Gonzalez, J. L. NavarreteBolanos, J. E. Botello-Alvarez, and J. Manuel Oliveros-Munoz, "Nonlinear homotopic continuation methods: a chemical engineering perspective review," Industrial \& Engineering Chemistry Research, vol. 52, no. 42, pp. 14729-14742, 2013.

[2] J. M. Oliveros-Munoz and H. Jimenez-Islas, "Hyperspherical path trackingmethodology as correction step in homotopic continuation methods," Chemical Engineering Science, vol. 97, pp. 413-429, 2013. 
[3] H. Jimenez-Islas, “Sehpe: programa para la solución de sistemas de ecuaciones no lineales mediante método homotópico con seguimiento hiperesférico," Avances en Ingeniería Química, vol. 6, pp. 174-179, 1996.

[4] D. J. Bates, J. D. Hauenstein, A. J. Sommese, and I. Wampler, "Adaptive multiprecision path tracking," SIAM Journal on Numerical Analysis, vol. 46, no. 2, pp. 722-746, 2008.

[5] D.-J. Bates, J.-D. Hauenstein, A.-J. Sommese, and C.-W. Wampler, "Stepsize control for adaptivemultiprecision path tracking," Contemporary Mathematics, vol. 496, pp. 21-31, 2009.

[6] A. Morgan and A. Sommese, "Computing all solutions to polynomial systems using homotopy continuation," Applied Mathematics and Computation, vol. 24, no. 2, pp. 115-138, 1987.

[7] H. Vazquez-Leal, R. Castaneda-Sheissa, A. Yildirim et al., "Biparameter homotopy-based direct current simulation of multistable circuits," Journal of Mathematics \& Computer Science, vol. 2, no. 3, pp. 137-150, 2012.

[8] A. Ushida, Y. Yamagami, Y. Nishio, I. Kinouchi, and Y. Inoue, "An efficient algorithm for finding multiple DC solutions based on the SPICE-oriented Newton homotopy method," IEEE Transactions on Computer-Aided Design of Integrated Circuits and Systems, vol. 21, no. 3, pp. 337-348, 2002.

[9] K. Ahuja, L. T. Watson, and S. C. Billups, "Probability-one homotopy maps for mixed complementarity problems," Computational Optimization and Applications, vol. 41, no. 3, pp. 363375, 2008.

[10] R. C. Melville, L. Trajkovic, S. Fang, and L. T. Watson, "Artificial parameter homotopy methods for the DC operating point problem," IEEE Transactions on Computer-Aided Design of Integrated Circuits and Systems, vol. 12, no. 6, pp. 861-877, 1993.

[11] H. Vázquez-Leal, L. Hernández-Martínez, A. Sarmiento-Reyes, R. Castãneda-Sheissa, and A. Gallardo-Del-Ángel, "Homotopy method with a formal stop criterion applied to circuit simulation," IEICE Electronics Express, vol. 8, no. 21, pp. 1808-1815, 2011.

[12] H. Vázquez-Leal, L. Hernández-Martínez, A. Sarmiento-Reyes, and R. Castañeda-Sheissa, "Numerical continuation scheme for tracing the double bounded homotopy for analysing nonlinear circuits," in Proceedings of the International Conference on Communications, Circuits and Systems, pp. 1122-1126, Hong Kong, China, May 2005.

[13] H. Vázquez-Leal, R. Castañeda-Sheissa, F. Rabago-Bernal, A. Sarmiento-Reyes, and U. Filobello-Niño, "Powering multiparameter homotopy-based simulation with a fast path-following technique," ISRN Applied Mathematics, vol. 2011, Article ID 610637, 7 pages, 2011.

[14] K. Yamamura, T. Sekiguchi, and Y. Inoue, "A fixed-point homotopy method for solving modified nodal equations," IEEE Transactions on Circuits and Systems I: Fundamental Theory and Applications, vol. 46, no. 6, pp. 654-665, 1999.

[15] L. Vandenberghe and J. Vandewalle, "Variable dimension algorithms for solving resistive circuits," International Journal of Circuit Theory and Applications, vol. 18, no. 5, pp. 443-474, 1990.

[16] R. Geoghegan, J. C. Lagarias, and R. C. Melville, “Threading homotopies and dc operating points of nonlinear circuits," SIAM Journal on Optimization, vol. 9, no. 1, pp. 159-178, 1999.

[17] D. M. Wolf and S. R. Sanders, "Multiparameter homotopy methods for finding dc operating points of nonlinear circuits," IEEE Transactions on Circuits and Systems. I. Fundamental Theory and Applications, vol. 43, no. 10, pp. 824-838, 1996.
[18] L. T. Watson and R. T. Haftka, "Modern homotopy methods in optimization," Computer Methods in Applied Mechanics and Engineering, vol. 74, no. 3, pp. 289-305, 1989.

[19] V. M. Pérez, J. E. Renaud, and L. T. Watson, "Homotopy curve tracking in approximate interior point optimization," Optimization and Engineering, vol. 10, no. 1, pp. 91-108, 2009.

[20] M. Kuno and J.-D. Seader, "Computing all real solutions to systems of nonlinear equations with a global fixed-point homotopy," Industrial \& Engineering Chemistry Research, vol. 27, no. 7, pp. 1320-1329, 1988.

[21] L. T. Watson, "Numerical linear algebra aspects of globally convergent homotopy methods," SIAM Review, vol. 28, no. 4, pp. 529-545, 1986.

[22] M. Sosonkina, L. T. Watson, and D. E. Stewart, "Note on the end game in homotopy zero curve tracking," ACM Transactions on Mathematical Software, vol. 22, no. 3, pp. 281-287, 1996.

[23] L. T. Watson, S. M. Holzer, and M. C. Hansen, "Tracking nonlinear equilibrium paths by a homotopy method," Nonlinear Analysis: Theory, Methods \& Applications, vol. 7, no. 11, pp. 12711282, 1983.

[24] L. T. Watson, "Globally convergent homotopy methods", in Encyclopedia of Optimization, C. A. Floudas and P. M. Pardalos, Eds., pp. 1272-1277, Springer, New York, NY, USA, 2009.

[25] L. T. Watson, S. C. Billups, and A. P. Morgan, "Algorithm 652: hompack: a suite of codes for globally convergent homotopy algorithms," Association for Computing Machinery. Transactions on Mathematical Software, vol. 13, no. 3, pp. 281-310, 1987.

[26] D. J. Bates, J. D. Hauenstein, and A. J. Sommese, "Efficient path tracking methods," Numerical Algorithms, vol. 58, no. 4, pp. 451459, 2011.

[27] E. L. Allgower and K. Georg, Numerical Path Following, 1994.

[28] E. L. Allgower and K. Georg, Continuation and Path Following, 1992.

[29] K. S. Gritton, J. D. Seader, and W. Lin, "Global homotopy continuation procedures for seeking all roots of a nonlinear equation," Computers \& Chemical Engineering, vol. 25, no. 7-8, pp. 1003-1019, 2001.

[30] L. Trajkovic, R.-C. Melville, and S.-C. Fang, "Passivity and nogain properties establish global convergence of a homotopy method for DC operating points," in Proceedings of the IEEE International Symposium on Circuits and Systems, vol. 2, pp. 914-917, New Orleans, La, USA, May 1990.

[31] J. Verschelde, "Polynomial homotopy continuation with phcpack," ACM Communications in Computer Algebra, vol. 44, no. 3-4, pp. 217-220, 2011.

[32] D. J. Bates, J. D. Hauenstein, A. J. Sommese, and C. W. Wampler, "Bertini: software for numerical algebraic geometry," https://bertini.nd.edu.

[33] T. L. Lee, T. Y. Li, and C. H. Tsai, "HOM4PS-2.0: a software package for solving polynomial systems by the polyhedral homotopy continuation method," Computing, vol. 83, no. 2-3, pp. 109-133, 2008.

[34] A. J. Sommese and C. W. Wampler II, The Numerical Solution of Systems of Polynomials: Arising in Engineering and Science, World Scientific, Singapore, 2005.

[35] T. Gunji, S. Kim, M. a. . Kojima, K. Fujisawa, and T. Mizutani, "PHoM-a polyhedral homotopy continuation method for polynomial systems," Computing. Archives for Scientific Computing, vol. 73, no. 1, pp. 57-77, 2004.

[36] H. Vazquez-Leal, A. Sarmiento-Reyes, Y. Khan et al., "New aspects of double bounded polynomial homotopy," British 
Journal of Mathematics \& Computer Science, vol. 3, no. 4, pp. 549-566, 2013.

[37] D. Torres-Munoz, H. Vazquez-Leal, L. Hernandez-Martinez, and A. Sarmiento-Reyes, "Improved spherical continuation algorithm with application to the double-bounded homotopy (dbh)," Computational and Applied Mathematics, vol. 33, no. 1, pp. 147-161, 2014.

[38] L. T. Watson, "Globally convergent homotopy algorithms for nonlinear systems of equations," Nonlinear Dynamics, vol. 1, no. 2, pp. 143-191, 1990.

[39] H. Vazquez-Leal, "Piece-wise-polynomial method," Computational \& Applied Mathematics, vol. 33, no. 2, pp. 289-299, 2014.

[40] I. Guerra-Gómez, T. McConaghy, and E. Tlelo-Cuautle, "Operating-point driven formulation for analog computer-aided design," Analog Integrated Circuits and Signal Processing, vol. 74, no. 2, pp. 345-353, 2013.

[41] R. Trejo-Guerra, E. Tlelo-Cuautle, V. H. Carbajal-Gomez, and G. Rodriguez-Gomez, "A survey on the integrated design of chaotic oscillators," Applied Mathematics and Computation, vol. 219, no. 10, pp. 5113-5122, 2013.

[42] R. Trejo-Guerra, E. Tlelo-Cuautle, J. M. Jimenez-Fuentes et al., "Integrated circuit generating 3- and 5-scroll attractors," Communications in Nonlinear Science and Numerical Simulation, vol. 17, no. 11, pp. 4328-4335, 2012.

[43] V. M. Jimenez-Fernandez, C. Reyes-Betanzo, M. AngelicaCerdan, Z. J. Hernandez-Paxtian, H. Vazquez-Leal, and A. Itzmoyotl-Toxqui, "Prediction of silicon dry etching using a piecewise linear algorithm," Journal of the Chinese Institute of Engineers, vol. 36, no. 7, pp. 941-950, 2013.

[44] V. M. Jimenez-Fernandez, E. Muñoz-Aguirre, H. Vazquez-Leal et al., "A piecewise linear fitting technique for multivalued twodimensional paths," Journal of Applied Research and Technology, vol. 11, no. 5, pp. 636-640, 2013.

[45] S. Pastore, "Fast and efficient search for all DC solutions of PWL circuits by means of oversized polyhedra," IEEE Transactions on Circuits and Systems. I. Regular Papers, vol. 56, no. 10, pp. 22702279, 2009.

[46] K. Yamamura and K. Yomogita, "Finding all solutions of piecewise-linear resistive circuits using an LP test," IEEE Transactions on Circuits and Systems I: Fundamental Theory and Applications, vol. 47, no. 7, pp. 1115-1120, 2000.

[47] L. Ying, S. Wang, and H. Xiaolin, "Finding all solutions of piecewise-linear circuits using mixed linear programming algorithm," in Proceedings of the Control and Decision Conference (CCDC '08), pp. 4204-4208, 2008, (Chinese).

[48] K. Yamamura and T. Ohshima, "Finding all solutions of piecewise-linear resistive circuits using linear programming," IEEE Transactions on Circuits and Systems I: Fundamental Theory and Applications, vol. 45, no. 4, pp. 434-445, 1998.

[49] K. Yamamura, "Finding all solutions of piecewise-linear resistive circuits using simple sign tests," IEEE Transactions on Circuits and Systems I: Fundamental Theory and Applications, vol. 40, no. 8, pp. 546-551, 1993.

[50] K. Yamamura and S. Tanaka, "Finding all solutions of piecewiselinear resistive circuits using the dual simplex method," in Proceedings of the IEEE Internaitonal Symposium on Circuits and Systems (ISCAS '00), vol. 4, pp. 165-168, Geneva, Switzerland, May 2000.

[51] M. Tadeusiewicz and S. Halgas, "Finding all the DC solutions of a certain class of piecewise-linear circuits," Circuits, Systems, and Signal Processing, vol. 18, no. 2, pp. 89-110, 1999.
[52] S. Pastore and A. Premoli, "Polyhedral elements: a new algorithm for capturing all the equilibrium points of piecewiselinear circuits," IEEE Transactions on Circuits and Systems I: Fundamental Theory and Applications, vol. 40, no. 2, pp. 124131, 1993.

[53] J. Katzenelson, "An algorithmfor solving nonlinear resistor networks," The Bell System Technical Journal, vol. 44, pp. 16051620, 1965.

[54] K. Yamamura and K. Horiuchi, "Globally and quadratically convergent algorithm for solving nonlinear resistive networks," IEEE Transactions on Computer-Aided Design of Integrated Circuits and Systems, vol. 9, no. 5, pp. 487-499, 1990.

[55] S. N. Stevens and P. M. Lin, "Analysis of piecewise-linear resistive networks using complementary pivot theory," IEEE Transactions on Circuits and Systems, vol. 28, pp. 429-441, 1981.

[56] J. T. J. van Eijndhoven, "Solving the linear complementarity problem in circuit simulation," SIAM Journal on Control and Optimization, vol. 24, no. 5, pp. 1050-1062, 1986.

[57] M. Tadeusiewicz and A. Kuczyński, "A very fast method for the DC analysis of diode-transistor circuits," Circuits, Systems, and Signal Processing, vol. 32, no. 2, pp. 433-451, 2013.

[58] L. O. Chua and S. M. Kang, "Canonical piecewise linear modeling," IEEE Transactions on Circuits and Systems, vol. 33, no. 5, pp. 511-525, 1984.

[59] W. Van Bokhoven, Piecewise Linear: Modeling and Analysis, Kluwer Technische Boeken, Deventer, The Netherlands, 1981.

[60] C. Guzelis and I. C. Goknar, "A canonical representation for piecewise-affine maps and its applications to circuit analysis," IEEE Transactions on Circuits and Systems, vol. 38, no. 11, pp. 1342-1354, 1991.

[61] L. Vandenberghe, B. L. de Moor, and J. Vandewalle, "The generalized linear complementarity problem applied to the complete analysis of resistive piecewise-linear circuits," IEEE Transactions on Circuits and Systems, vol. 36, no. 11, pp. 1382-1391, 1989.

[62] T. A. M. Kevenaar and D. M. W. Leenaerts, "A comparison of piecewise linearmodel descriptions," IEEE Transactions on Circuits and Systems I: Fundamental Theory and Applications, vol. 38, no. 12, pp. 996-1004, 1992.

[63] C. van de Panne, "A complementary variant of Lemke's method for the linear complementary problem," Mathematical Programming, vol. 7, pp. 283-310, 1974.

[64] L. O. Chua and R. L. P. Ying, "Finding all solutions of piecewiselinear circuits," International Journal of Circuit Theory and Applications, vol. 10, no. 3, pp. 201-229, 1982.

[65] K. Yamamura and M. Ochiai, "An efficient algorithm for finding all solutions of piecewise-linear resistive circuits," IEEE Transactions on Circuits and Systems I: Fundamental Theory and Applications, vol. 39, no. 3, pp. 213-221, 1992.

[66] K. Yamamura, "Simple algorithms for tracing solution curves," IEEE Transactions on Circuits and Systems I: Fundamental Theory and Applications, vol. 40, no. 8, pp. 537-541, 1993.

[67] D. Niu, X. Wu, Z. Jin, and Y. Inoue, "An effective and globally convergent newton fixedpoint homotopy method for mos transistor circuits," IEICE TRANSACTIONS on Fundamentals of Electronics, Communications and Computer Sciences, vol. 96, no. 9, pp. 1848-1856, 2013.

[68] J. Lee and H. Chiang, "Constructive homotopy methods for finding all or multiple dc operating points of nonlinear circuits and systems," IEEE Transactions on Circuits and Systems I: Fundamental Theory and Applications, vol. 48, no. 1, pp. 35-50, 2001. 

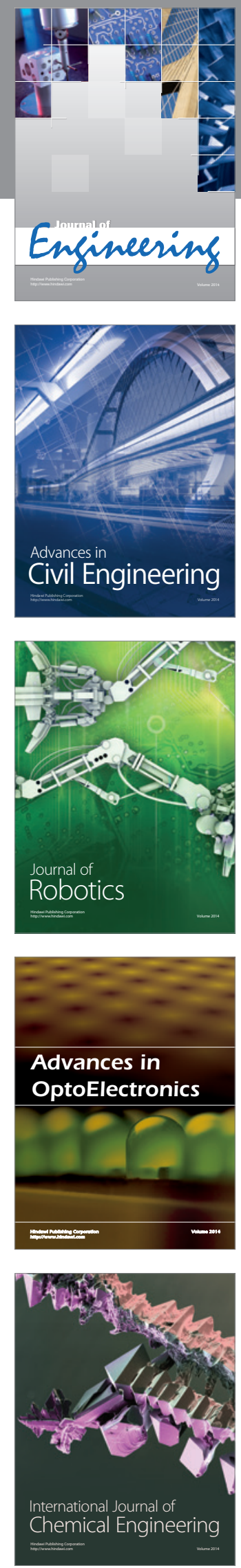

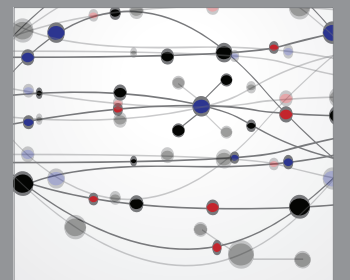

The Scientific World Journal
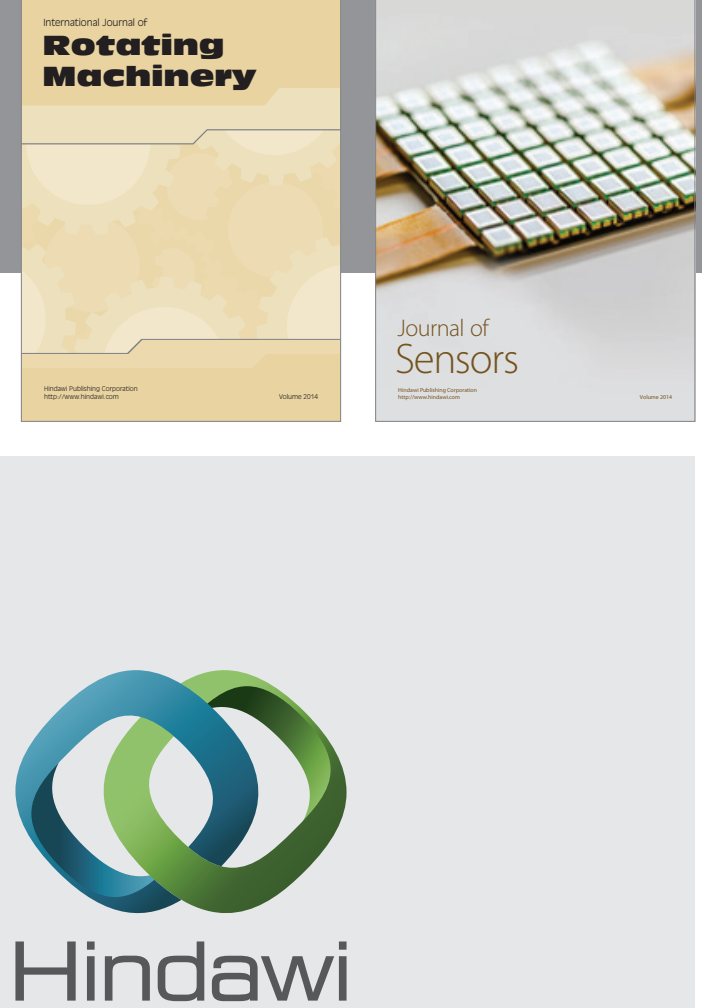

Submit your manuscripts at http://www.hindawi.com
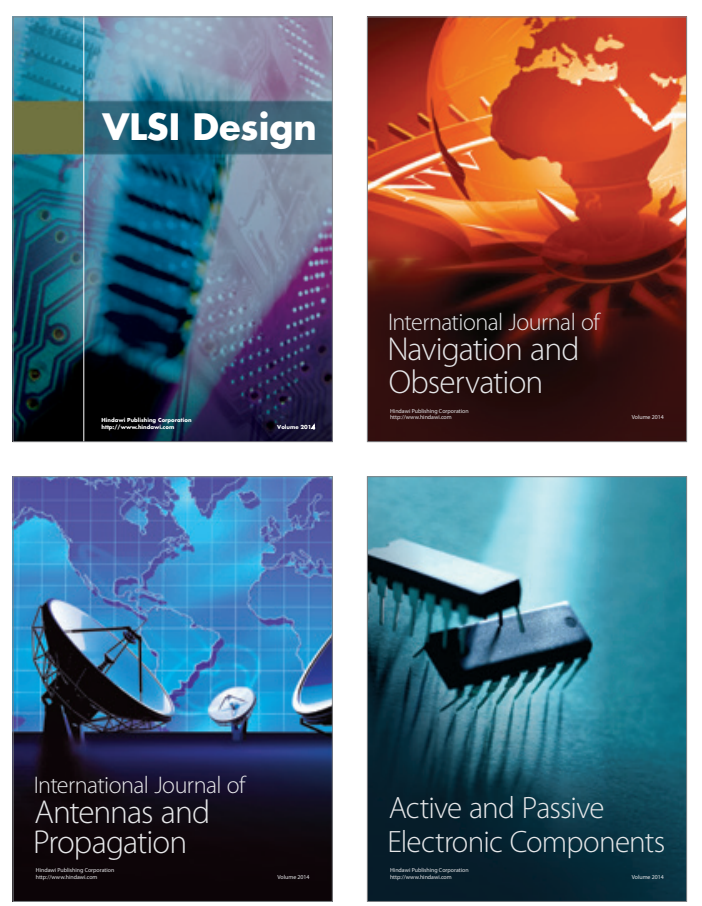
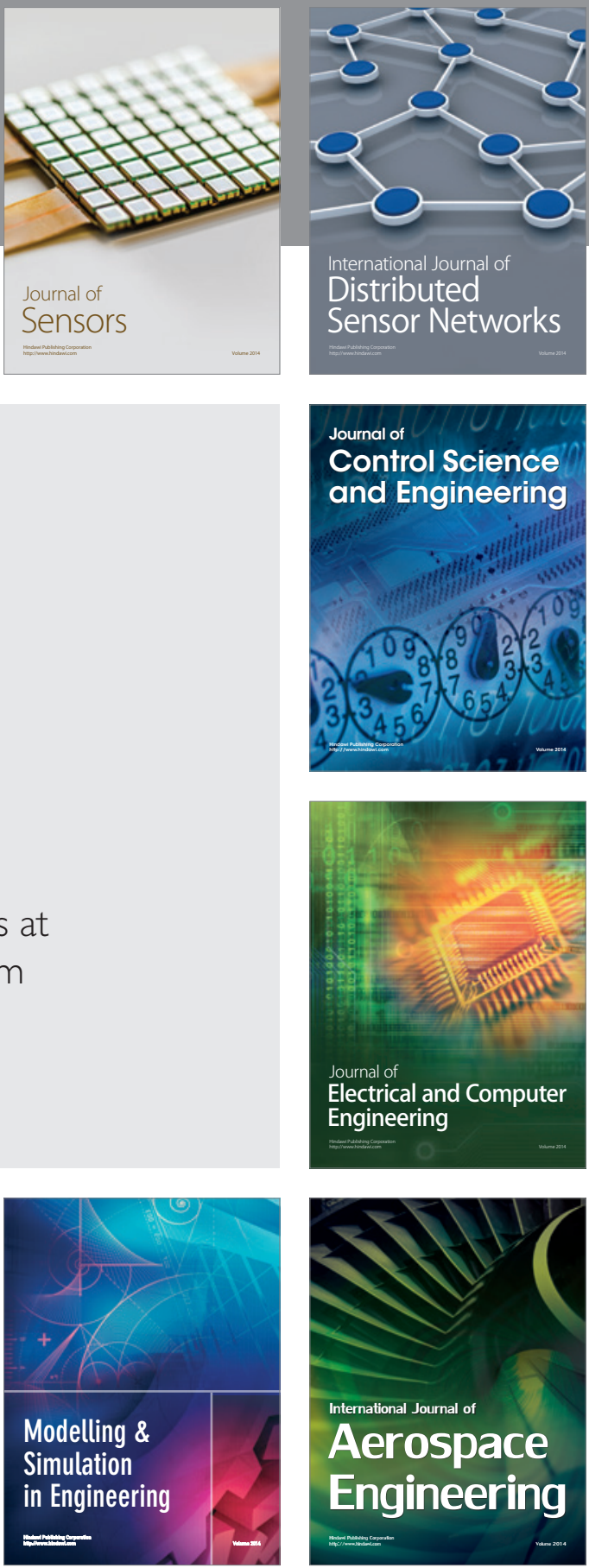

Journal of

Control Science

and Engineering
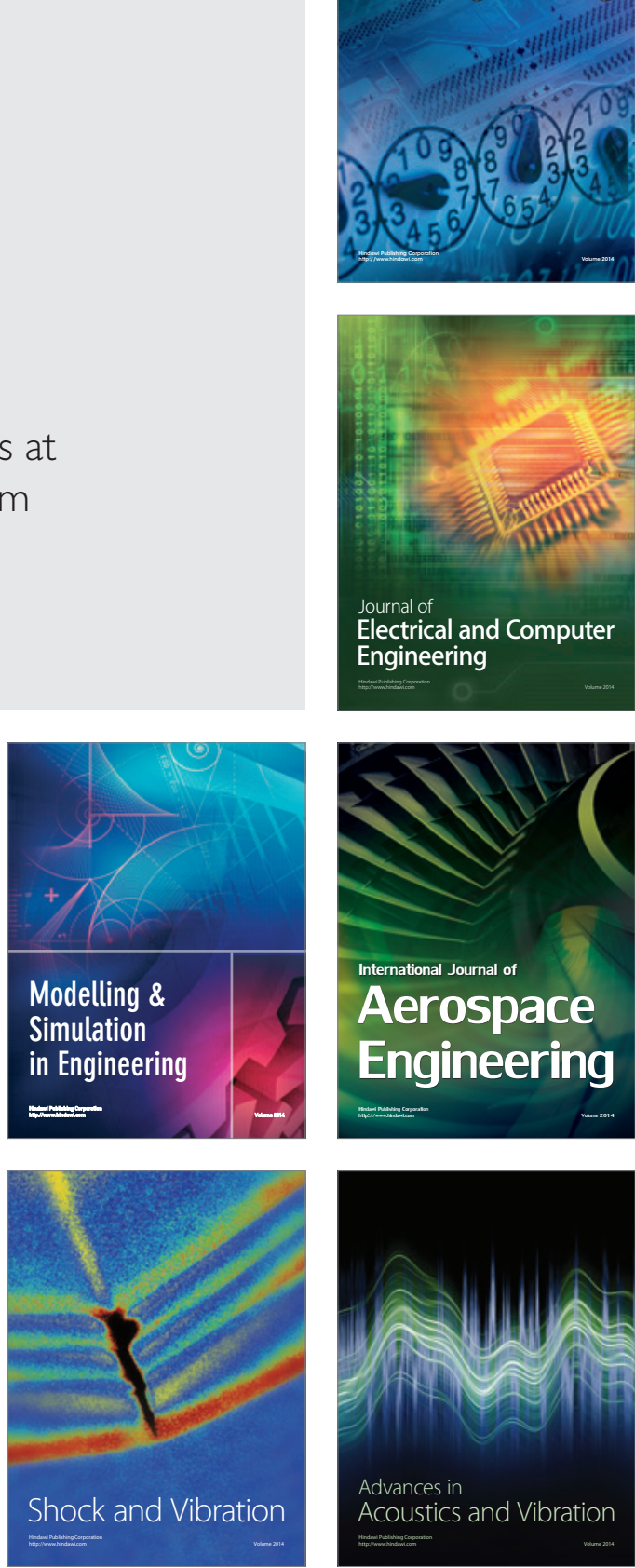